\title{
-NOTES-
}

\section{A SUGGESTED MODIFICATION OF NOISE THEORY*}

\section{BY JULIAN KEILSON (Massachusetts Institute of Technology)}

Abstract. A class of stationary, equilibrium, Markoff processes is demonstrated all of which have the same equilibrium distribution, $W_{0}(x)$, and correlation function $R(t)=E_{0} \exp \left(-t / \tau_{0}\right)$ differing from each other in the number of zero crossings of the system per second. The processes are described by an integral equation characterized by a parameter $\gamma$. As $\gamma$ approaches 1, the integral equation passes over into the FokkerPlanck equation

$$
\tau_{0} \frac{\partial W}{\partial t}=E_{0} \frac{\partial^{2} W}{\partial x^{2}}+\frac{\partial}{\partial x}(x W) .
$$

Since the number of zero crossings per second of the system becomes infinite as $\gamma$ goes to one, the degenerate nature of the Fokker-Planck process is made evident.

1. Introduction. Any stationary Markoffian motion of a system in one dimension may be described by an equation of the form

$$
\frac{\partial W(x, t)}{\partial t}=-W(x, t) \int A\left(x, x^{\prime}\right) d x^{\prime}+\int W\left(x^{\prime}, t\right) A\left(x^{\prime}, x\right) d x^{\prime},
$$

where $W(x, t) d x$ is the probability of finding the system in the interval $(x, x+d x)$ at time t. $A\left(x, x^{\prime}\right) d x^{\prime}$ is the probability per unit time that the system if at $x$, will jump to the interval $\left(x^{\prime}, x^{\prime}+d x^{\prime}\right)$. Equation (1) describes the manner in which changes in local probability density can occur. It is seen that

$$
\frac{d}{d t} \int W(x, t) d x=0
$$

so that probability is conserved.

The stationary Markoff character of the motion is assured by the formulation in terms of a time independent transition function $A\left(x, x^{\prime}\right)$. By stationary Markoff is meant that if at $t=0$, the system is at $x_{0}$, its subsequent distribution is completely described by the second order conditional probability:

$$
W(x, t)=P_{2}\left(x_{0} \mid x ; t\right) .
$$

For some $A\left(x, x^{\prime}\right)$ the process will be an equilibrium process in the sense that

$$
\lim _{t \rightarrow \infty} P_{2}\left(x_{0} \mid x ; t\right)=W_{0}(x)
$$

is independent of $x_{0}$.

If $A\left(x, x^{\prime}\right)$ is not localized about $x=x^{\prime}$ the stochastic motion described is discontinuous. The system jumps about from point to point. As $A\left(x, x^{\prime}\right)$ becomes more localized about $x=x^{\prime}$, the jumps become on the average smaller, i.e., the motion becomes "more continuous".

*Received April 7, 1953. The research in this document was supported jointly by the Army, Navy and Air Force under contract with the Massachusetts Institute of Technology. 
In this paper a class of transition function $A_{\gamma}\left(x, x^{\prime}\right)$ will be studied. It will be shown that, as $\gamma \rightarrow 1$ and $A_{\gamma}\left(x, x^{\prime}\right)$ becomes increasingly localized about $x=x^{\prime}$, the integral equation (1) passes over into the Fokker-Planck equation

$$
\tau_{0} \frac{\partial W}{\partial t}=E_{0} \frac{\partial^{2} W}{\partial x^{2}}+\frac{\partial}{\partial x}(x W) .
$$

The nature of the motion described by the Fokker-Planck equation as indicated by this limiting process will be discussed, and the physical implications of this study examined.

2. Properties of the integral equation. Consider the process described by the transition function

$$
A\left(x, x^{\prime}\right)=\frac{1}{T_{m f}}(\beta / \pi)^{1 / 2} \exp \left[-\beta\left(x^{\prime}-\gamma x\right)^{2}\right] .
$$

Here, $T_{m f}$, the mean free time of the system, is independent of $x$, i.e.

$$
\int_{-\infty}^{+\infty} A\left(x, x^{\prime}\right) d x^{\prime}=1 / T_{m s}
$$

is the mean number of transitions of the system per unit time; $\beta$ describes the dispersion occurring at each transition. The larger $\beta$, the more "continuous" is the motion; $\gamma$ is a parameter describing the relaxation or damping of the system, i.e. if $\langle x\rangle$ is the mean value of $x$,

$$
\langle x\rangle=\int x W(x, t) d x
$$

then

$$
\frac{d\langle x\rangle}{d t}=\frac{-\langle x\rangle}{T_{m f} /(1-\gamma)}
$$

as is readily deduced from Eq. (1). The decay time of system is, then

$$
\tau=T_{m f} /(1-\gamma)
$$

The closer $\gamma$ is to one, the smaller is the damping and the longer the decay time. $\tau$ is also the correlation time of the system, for

$$
R(t)=\int W_{0}\left(x_{0}\right) x_{0} P_{2}\left(x_{0} \mid x ; t\right) x d x_{0} d x .
$$

Since from (9) $\langle x\rangle=x_{0} \exp (-t / \tau)$, we have

$$
R(t)=\int W_{0}\left(x_{0}\right) x_{0}^{2} \exp (-t / \tau) d x_{0},
$$

i.e.

$$
R(t)=E \exp (-t / \tau)
$$

where

$$
E=\int W_{0}(x) x^{2} d x
$$


Equation (1) with $A\left(x, x^{\prime}\right)$ given by (6) may be solved in the following way. Use is made of the expansion

$$
\begin{gathered}
\exp \left[-(x-\gamma y)^{2} /\left(1-\gamma^{2}\right)\right]=\left\{\left[\pi\left(1-\gamma^{2}\right)\right]^{1 / 2} \exp \left[+\left(y^{2}-x^{2}\right) / 2\right]\right\} \\
\sum_{0}^{\infty} \gamma^{n} \psi_{n}(x) \psi_{n}(y),
\end{gathered}
$$

where $\psi_{n}(x)$ is the set of orthonormal Hermite functions

$$
\psi_{n}(x)=\left(\frac{1}{2^{n} n ! \pi^{1 / 2}}\right)^{1 / 2} \exp \left(x^{2} / 2\right)(-d / d x)^{n} \exp \left(-x^{2}\right) .
$$

Then

$$
A\left(x^{\prime}, x\right)=\frac{\alpha}{T_{m}} \exp \left[-\frac{\alpha^{2}}{2}\left(x^{2}-x^{\prime 2}\right)\right] \sum_{0}^{\infty} \gamma^{n} \psi_{n}(\alpha x) \psi_{n}\left(\alpha x^{\prime}\right)
$$

where

$$
\alpha=\left[\beta\left(1-\gamma^{2}\right)\right]^{1 / 2} .
$$

In terms of

$$
\phi(x, t)=\exp \left[\frac{\alpha^{2}}{2} x^{2}\right] W(x, t)
$$

Eq. (1) becomes

$$
\frac{\partial \phi}{\partial t}=-\frac{\phi}{T_{m f}}+\frac{\alpha}{T_{m s}} \int \sum_{0}^{\infty} \psi_{n}(\alpha x) \psi_{n}\left(\alpha x^{\prime}\right) \gamma^{n} \phi\left(x^{\prime}, t\right) d x^{\prime} .
$$

If we expand $\phi$ in terms of the orthonormal set $\alpha^{1 / 2} \psi_{n}(\alpha x)$

$$
\phi(x, t)=\sum_{0}^{\infty} a_{n} \alpha^{1 / 2} \psi_{n}(\alpha x),
$$

our equation separates into

$$
\frac{d a_{n}}{d t}=-\frac{a_{n}}{T_{m}}+\frac{\gamma^{n} a_{n}}{T_{m}}
$$

If, at $t=0$,

$$
W(x, 0)=\delta\left(x-x_{0}\right)=\sum_{0}^{\infty} \alpha \psi_{n}(\alpha x) \psi_{n}\left(\alpha x_{0}\right),
$$

then

$$
a_{n}(0)=\alpha^{1 / 2} \exp \left(\frac{\alpha^{2}}{2} x_{0}^{2}\right) \psi_{n}\left(\alpha x_{0}\right)
$$

and

$$
P_{2}\left(x_{0} \mid x ; t\right)=\exp \left[\frac{\alpha^{2}}{2}\left(x_{0}^{2}-x^{2}\right)\right] \sum_{0}^{\infty}\left\{\alpha \psi_{n}(\alpha x) \psi_{n}\left(\alpha x_{0}\right) \exp \left[-t\left(1-\gamma^{n}\right) / T_{m f}\right]\right\}
$$


We see that

$$
\begin{aligned}
\lim _{t \rightarrow \infty} P_{2}\left(x_{0} \mid x ; t\right) & =W_{0}(x)=\pi^{-1 / 2} \alpha \exp \left(-\alpha^{2} x^{2}\right) \\
& =\pi^{-1 / 2}\left[\beta\left(1-\gamma^{2}\right)\right]^{1 / 2} \exp \left[-\beta\left(1-\gamma^{2}\right) x^{2}\right]
\end{aligned}
$$

and

$$
E=\left\langle x^{2}\right\rangle=\left[2 \beta\left(1-\gamma^{2}\right)\right]^{-1}
$$

3. Passage to the Fokker-Planck process. The Fokker-Planck equation, (5), contains two parameters, the correlation time $\tau_{0}$ and the equilibrium mean square, $E_{0}=\left\langle x^{2}\right\rangle$.

Our transition function $A\left(x, x^{\prime}\right)$ contains three variables $\tau, E, \gamma$. Let us maintain the values $\tau=\tau_{0}$, and $E=E_{0}$ and permit $\gamma$ to pass through a set of values approaching one. To do so we adjust $\mathrm{T}_{m f}$ and $\beta$ to the value of $\gamma$ through the equations

$$
T_{m f}=\tau_{0}(1-\gamma)
$$

and

$$
\beta=\frac{1}{2 E_{0}\left(1-\gamma^{2}\right)} \simeq \frac{1}{4 E_{0}(1-\gamma)}
$$

These parameter values define a set of processes $A_{\gamma}$ all of which will have the same equilibrium distribution and the same correlation function as the corresponding FokkerPlanck process.

Indeed, the corresponding Fokker-Planck process is just the limit of the process $A_{\gamma}$ as $\gamma \rightarrow 1$.

This may be seen in two ways. First one may pass to the limit $\gamma=1$ in the conditional probability function.

Since for the process $A_{\gamma}$

$$
\alpha=\left(\frac{E_{0}}{2}\right)^{1 / 2}, \quad \lim \frac{1-\gamma^{n}}{T_{m f}}=\frac{n}{\tau_{0}}
$$

we have

$$
\begin{aligned}
\lim _{\gamma \rightarrow 1} P_{2 \gamma}\left(x_{0} \mid x ; t\right) & =\exp \left[\frac{\alpha^{2}}{2}\left(x_{0}^{2}-x^{2}\right)\right] \sum_{0}^{\infty}\left\{\alpha \psi_{n}(\alpha x) \psi_{n}\left(\alpha x_{0}\right) \exp \left(-n t / \tau_{0}\right)\right. \\
& =\left\{2 E_{0} \pi\left[1-\exp \left(-2 t / \tau_{0}\right)\right]\right\}^{-1 / 2} \exp \left\{\frac{-\left[x-x_{0} \exp \left(-t / \tau_{0}\right)\right]^{2}}{2 E_{0}\left[1-\exp \left(-2 t / \tau_{0}\right)\right]}\right\}
\end{aligned}
$$

and this is indeed the second order conditional probability of our equation (5).

It can also be seen directly that the integral equation passes over into the FokkerPlanck equation.

The integral equation can always be rewritten formally* as a partial differential equation of infinite order

$$
\frac{\partial W(x, t)}{\partial t}=\sum_{1}^{\infty} \frac{\partial^{n}}{\partial x^{n}}\left(A_{n}(x) W(x, t)\right),
$$

*See p. 246 Keilson and Storer, Q. Appl. Math. 10, 243-253 (1952). 
where

$$
A_{n}(x)=\frac{1}{n !} \int\left(x-x^{\prime}\right)^{n} A\left(x, x^{\prime}\right) d x^{\prime}
$$

In the limit $\gamma \rightarrow 1, A_{1}(x)$ and $A_{2}(x)$ do not vanish, but all higher moments do vanish. It is readily found that

$$
A_{1}(x)=x / \tau_{0}
$$

and

$$
A_{2}(x)=\frac{(1-\gamma)^{2} x^{2}}{2 T_{m f}}+\frac{\left(1-\gamma^{2}\right) E_{0}}{2 T_{m f}} \rightarrow \frac{E_{0}}{\tau_{0}}
$$

The higher moments

$$
A_{n}(x)=\frac{1}{n !} \int\left\{(1-\gamma) x+\left(\gamma x-x^{\prime}\right)\right\}^{n} \frac{1}{T_{m f}}\left(\frac{\beta}{\pi}\right)^{1 / 2} \exp \left[-\beta\left(x^{\prime}-\bar{\gamma} x\right)^{2}\right] d x^{\prime}
$$

contain terms

$$
(1-\gamma)^{m} x^{m}\left\langle\left(\gamma x-x^{\prime}\right)^{p}\right\rangle,
$$

where either $m>2$ or $p>2$. A simple examination reveals that all such terms go to zero as $\gamma$ approaches one.

4. Zero crossings of the system $A_{\gamma}$. It is easily seen that the number of times per second that the system with its motion characterized by $A\left(x, x^{\prime}\right)$ will cross zero is given by

$$
\begin{aligned}
j_{+}(0) & =\int_{-\infty}^{0} W_{0}(x) d x \int_{0}^{\infty} A\left(x, x^{\prime}\right) d x^{\prime} \\
& =\frac{\left(1-\gamma^{2}\right)^{1 / 2}}{\pi T_{m f}} \int_{-\infty}^{0} \int_{0}^{\infty} \exp \left[-x^{2}-y^{2}-2 \gamma x y\right] d x d y \\
& =\frac{\left(1-\gamma^{2}\right)^{1 / 2}}{\pi T_{m f}} \int_{A} \exp \left[-S^{2}(1+\gamma)-T^{2}(1-\gamma)\right] d s d t,
\end{aligned}
$$

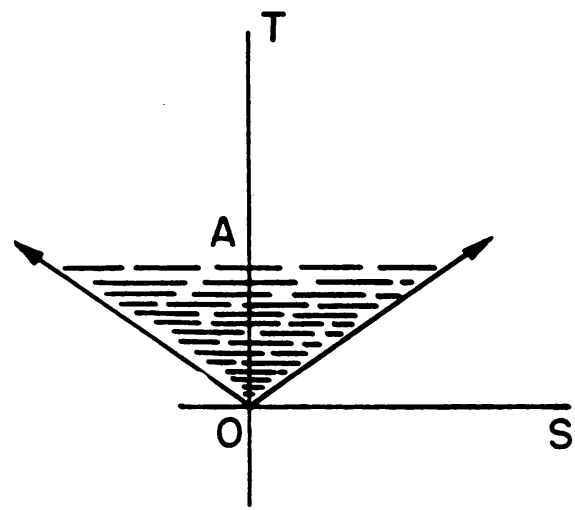

Fig. 1 
where $A$ is the shaded area shown in Fig. 1, i.e.

$$
j_{+}(0)=\frac{1}{\pi T_{m f}} \tan ^{-1}\left\{\left(\frac{1-\gamma}{1+\gamma}\right)^{1 / 2}\right\} .
$$

For the process $A_{\gamma}$,

$$
j_{+}(0)=\frac{1}{\pi \tau_{0}(1-\gamma)} \tan ^{-1}\left\{\left(\frac{1-\gamma}{1+\gamma}\right)^{1 / 2}\right\} .
$$

As $\gamma$ approaches one, $j_{+}(0)$ becomes infinite. This will be true not only for zero but for all $x$. The implication is plain. The Fokker-Planck process is a degenerate process in which the one sided current density of the system is infinite. A Fokker-Planck model for the velocity motion of a colloid particle would describe an infinite number of changes of direction of the particle per unit time. Such a model used to describe voltage fluctuations would imply an infinite number of polarity reversals per second. Since a process $A \gamma$ will afford the same correlation function and equilibrium distribution, and finite polarity reversal frequency, it is suggested that such a model may better describe noise, and that the number of zero crossings be regarded as an independent macroscopic physical quantity on an equal footing with $\tau_{0}, E_{0}$.

The author thanks Dr. Franz Stumpers of Phillips Eindhoven and Dr. Edwin Akutowicz for their interest and encouragement.

\section{EVALUATION OF CONSTANTS IN CONFORMAL REPRESENTATION*}

By SAMUEL I. PLOTNICK ANd THOMAS C. BENTON (Pennsylvania State University)

In using the Schwarz-Christoffel transformation [1],

$$
d z=K \prod_{i=1}^{n}\left(\zeta-\zeta_{i}\right)^{\left(\alpha_{i} / \pi\right)-1} d \zeta=K f(\zeta) d \zeta
$$

whereby the upper half $\zeta$-plane is mapped into a simple connected polygon, the evaluation of the unknown constant $K$ (if complex $K=c e^{i \lambda}, c, \lambda$ real), is oftentimes tedious. We shall show a simple method of evaluating the unknown constant $K$ by examples, proving first a

TheоRem: By the Schwarz-Christoffel transformation if $\zeta_{i}$ in the $\zeta$-plane corresponds to two points $P_{i}, Q_{i}$ in the z-plane and $\zeta=\zeta_{i}$ is a simple pole of $f(\zeta)$, then

$$
K=\frac{\operatorname{dist}\left(P_{i}, Q_{i}\right)}{\pi i R\left(\zeta=\zeta_{i}\right)}
$$

$R$, denoting residue and dist $\left(P_{i}, Q_{i}\right)$, denoting the distance between the two points $P_{i}$ and $Q_{i}$.

${ }^{*}$ Received May 8, 1953. 